\title{
The oral-aboral axis of a sea urchin embryo is specified by first cleavage
}

\author{
R. ANDREW CAMERON ${ }^{1}$, SCOTT E. FRASER ${ }^{2}$, ROY J. BRITTEN ${ }^{1}$ and ERIC H. DAVIDSON ${ }^{1}$ \\ ${ }^{1}$ Division of Biology 156-29, California Institute of Technology, Pasadena, California 91125, USA \\ ${ }^{2}$ Department of Physiology and Biophysics, University of California, Irvine, Irvine, California 92717, USA
}

\begin{abstract}
Summary
Several lines of evidence suggest that the oral-aboral axis in Strongylocentrotus purpuratus embryos is specified at or before the 8-cell stage. Were the oral-aboral axis specified independently of the first cleavage plane, then a random association of this plane with the blastomeres of the four embryo quadrants in the oral-aboral plane (viz. oral, aboral, right and left) would be expected. Lineage tracer dye injection into one blastomere at the 2-cell stage and observation of the resultant labeling patterns demonstrates instead a strongly nonrandom association. In at least ninety percent of cases, the progeny of the aboral blastomeres are associated
\end{abstract}

with those of the left lateral blastomeres and the progeny of the oral blastomeres with the right lateral ones, respectively. Thus, ninety percent of the time the oral pole of the future oral-aboral axis lies $45^{\circ}$ clockwise from the first cleavage plane as viewed from the animal pole. The nonrandom association of blastomeres after labeling of the 2-cell stage implies that there is a mechanistic relation between axis specification and the positioning of the first cleavage plane.

Key words: iontophoretic injection, Strongylocentrotus purpuratus, cell lineage.

\section{Introduction}

As in most deuterostome examples, only one of the two embryonic axes in the eggs of regularly developing sea urchins appears to be fixed prior to fertilization. The initial axis is the animal-vegetal ( $\mathrm{A}-\mathrm{V})$ axis, which is structured in the cytoskeletal organization of the unfertilized egg (Boveri, 1901; Schroeder, 1980a,b; Sardet \& Chang, 1985). The radial position of the oral-aboral $(\mathrm{O}-\mathrm{Ab})$ axis, which lies in an approximately horizontal plane with respect to the (conventionally) vertical A-V axis, is certainly not irreversibly established prior to fertilization. Thus this axis can be respecified under numerous circumstances, e.g. when separate meridional quarter embryos all develop into complete pluteus larvae, or when meridional half eggs or embryos are fused, and subsequently develop into normal pluteus larvae (reviewed by Hörstadius, 1973, pp. 96-102). Nor does the position of the $\mathrm{O}-\mathrm{Ab}$ axis display any particular relation to the point of sperm entry (early data reviewed by Hörstadius, 1973, p. 96; Schroeder, 1980a; our unpublished observations). However, there is good evidence that in at least some sea urchin species the $\mathrm{O}-\mathrm{Ab}$ axis has been specified as early as third cleavage. Czihak (1963) showed that by this stage in Paracentrotus lividus embryos the blastomeres of the animal quartet that will contribute the facial oral ectoderm of the future embryo can be distinguished by a polarized accumulation of redox enzyme, detectable by cytological stains. Recently, Cameron et al. (1987) demon- strated by injection of a fluoresceinated dextran lineage tracer into single blastomeres of 8-cell Strongylocentrotus purpuratus embryos that one blastomere of the animal blastomere quartet (termed $\mathbf{N a}$ ) gives rise exclusively to a central patch of aboral ectoderm, while the diametrically opposing blastomere (No) gives rise exclusively to the facial ectoderm. The two lateral blastomeres of this quartet ( $\mathbf{N L}_{\text {Lent }}$ and $\mathbf{N L}_{\mathbf{R i g h t}}$ ) contribute to both oral and aboral ectodermal structures (these respective fates are segregated into separate clones two cleavages later; Cameron et al. 1989). The ectodermal derivatives of the vegetal quartet of 8-cell embryos (i.e.

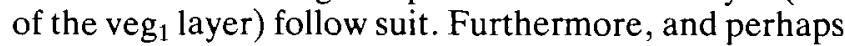
most importantly, the oral-aboral axis thus demonstrable at 3rd cleavage establishes the ultimate plane of bilateral symmetry of the completed pluteus larva (the actual axis of bilateral symmetry in the pluteus, i.e. the line from mouth to aboral vertex, is not coincident with the original $\mathrm{O}-\mathrm{Ab}$ axis because of the distortion occurring in this plane after gastrulation; see Fig. 10 of Cameron et al. 1987). Thus the ultimate clonal contributions of the $\mathbf{N a}, \mathbf{N o}$, and the $\mathbf{N L}_{\mathbf{L}}$ and $\mathbf{N L}_{\mathbf{R}}$ blastomeres are bilaterally symmetric with respect to the larval body plan. In the sea urchin embryo, the first two cleavage planes are vertical and orthogonal to one another, their intersection corresponding to the preformed A-V axis. The results of Cameron et al. (1987) required that the radial positions of the $\mathrm{O}-\mathrm{Ab}$ axis lie equidistant from these first two planes of cleavage; i.e. if viewed from the animal pole, at an angle of about $45^{\circ}$ 
from each of the first two cleavage furrows (coincident with the fourth cleavage furrow, bisecting the No and $\mathrm{Na}$ blastomeres). In summary, the cytological polarization that indicates the frame line of bilateral symmetry by third cleavage lies about $45^{\circ}$ from the initial two cleavage furrows. This raises the possibility that there may exist a causal relation between the establishment of the initial cleavage planes and the specification of the $\mathrm{O}-\mathrm{Ab}$ axis.

In this paper, we focus specifically on the relation between the positions of the $\mathrm{O}-\mathrm{Ab}$ axis and the plane of first cleavage, utilizing the invariant lineage of the pluteus ectoderm revealed in our prior study (Cameron et al. 1987). The fluoresceinated dextran tracer was injected into one of the first two blastomeres, and the pattern of ectodermal labeling at the pluteus stage was analyzed with respect to the known contributions of the various 8-cell-stage blastomeres. Fig. 1 illustrates diagrammatically the four possible ectodermal labeling patterns that would result at the pluteus stage, from dye injection into either of the first two blastomeres. Two of these cases result if the $\mathrm{O}-\mathrm{Ab}$ axis passes $45^{\circ}$ clockwise from the plane of first cleavage (as viewed from the animal pole) and is oriented so that the cleavage plane forms the boundary between the No and the left NL blastomeres (Fig. 1A,B); the other two cases result if the $\mathrm{O}-\mathrm{Ab}$ axis passes $45^{\circ}$ counterclockwise from the plane of first cleavage, and this plane forms the boundary between the No and the right NL blastomeres. Were specification of the $\mathrm{O}-\mathrm{Ab}$ axis to occur prior to first cleavage, but independently of the establishment of the plane of first cleavage, or were specification of the $\mathrm{O}-\mathrm{Ab}$ axis only to occur later in cleavage, all four cases would be expected to occur with equal frequency. We find, however, that one complementary pair of these labeling patterns is obtained in at least $90 \%$ of cases, illustrated in Fig. 1A,B, the alternative pair of labeling patterns occurs only rarely. Thus the $\mathrm{O}-\mathrm{Ab}$ axis is evidently specified in a predictable location and orientation with respect to the plane of first cleavage.

\section{Materials and methods}

\section{Embryo culture and preparation for injection}

Sea urchin embryos were prepared for iontophoretic injection using a modification of the methods employed for pressure injection (Cameron et al. 1987). Briefly, Strongylocentrotus purpuratus gametes were obtained by injection of $0.5 \mathrm{M}-\mathrm{KCl}$ into the perivisceral coelom. Eggs were collected in $0.45 \mu \mathrm{m}$ filtered natural sea water with $1 \mathrm{~mm}$-3-amino, 1,2,4-triazole (ATA) (Sigma, Inc.) added, to inhibit hardening of the fertilization envelope. Eggs were dejellied in $\mathrm{pH} 5.0$ sea water and semen was kept undiluted on ice. Eggs were electrostatically fixed to $60 \mathrm{~mm}$ Petri dish lids treated with $1 \%$ protamine sulfate (McMahon et al. 1985). The eggs were fertilized with a freshly diluted sperm suspension and the embryos allowed to develop to the 2-cell stage. Typically $10-12$ eggs were attached to a single dish and about 20 dishes were prepared in a single experiment. All sea waters used through the incubation to 2-cell stage contained $1 \mathrm{~mm}$-ATA to prevent hardening of the fertilization envelope.

Just before injection, the embryos were rinsed three times in calcium- and magnesium-free sea water (CMFSW) to soften the tough extracellular coats and facilitate penetration with microelectrodes. Sometimes, 2.5 to $5 \mathrm{~mm}$-EDTA was added to this solution to further reduce divalent cation levels. Following injection, calcium and magnesium ion concentrations were returned to those of artificial sea water, the dish was then washed several times with $0.45 \mu \mathrm{m}$ filtered sea water containing penicillin (20units $\mathrm{ml}^{-1}$ ) and streptomycin $\left(50 \mu \mathrm{g} \mathrm{ml}^{-1}\right)$ and the embryos were incubated in the dark for $72 \mathrm{~h}$ at $16^{\circ} \mathrm{C}$.

\section{Fluorescent dye injection and analysis}

Single blastomeres of 2-cell embryos were impaled with thinwalled aluminosilicate glass microelectrodes possessing tip resistances in the range of $10-50$ megohms. The electrodes were filled at the tip with $100 \mathrm{mg} \mathrm{ml}^{-1}$ of the fixable lineage tracer, lysyl rhodamine dextran (Molecular Probes), and backfilled with $1.2 \mathrm{M}-\mathrm{LiCl}$. Fluorescent dextran was ejected with positive current of 5 to $10 \mathrm{nA}$ for $20-60 \mathrm{~s}$. Lucifer Yellow $\mathrm{CH}$ (Molecular Probes) at a concentration of $3 \%$ was ejected from similarly back-filled microelectrodes with negative current of 2 to $5 \mathrm{nA}$ for 10 to $30 \mathrm{~s}$. Whole mounts of live plutei or plutei fixed in 1-2\% formaldehyde in sea water were recorded on Kodak Ektachrome film directly or on videotape through a light-intensifying camera (SIT) attached to an image processing system (Imaging Technologies).

\section{Results}

Labeling patterns observed in pluteus-stage embryos Embryos were raised from eggs injected at the 2-cell stage with rhodamine dextran as described, and the spatial patterns of epifluorescence in the ectoderm were carefully examined at the pluteus stage. These were classified according to the lineage contributions expected according to the results of Cameron et al. (1987). These ectodermal labeling patterns, which derive from the injection of individual blastomeres at the 8-cell stage, are contiguous, uniquely positioned patches of cells each of which are descended from a specific progenitor. This characteristic of ectodermal cell lineage, which serves as a basis for the present analysis, derives from two features of sea urchin development: (1) an invariant pattern of cleavage in undisturbed embryos leading to the five tissue territories of the pluteus; and (2) the totally nonmotile behavior of embryonal ectoderm cells up to and during the distortion of shape leading to the pluteus form. Except for a few abnormally developing embryos (these usually contained undivided labeled cells in the blastocoel and were excluded from the sample considered), the pattern of contributions expected from this earlier study provided a consistent guide for interpretation. In no case were the expected boundaries between labeled and unlabeled regions violated. The patterns observed thus conformed explicitly to those predicted in the diagrams shown in Fig. 1. Examples of the pair of complementary patterns observed in all but a small minority of cases are shown in Figs 2 and 3. It should be noted that, although we are here concerned only with fluorescence in the ectoderm, the archenteron in these embryos is also labeled, and it is difficult to find a single focal plane 

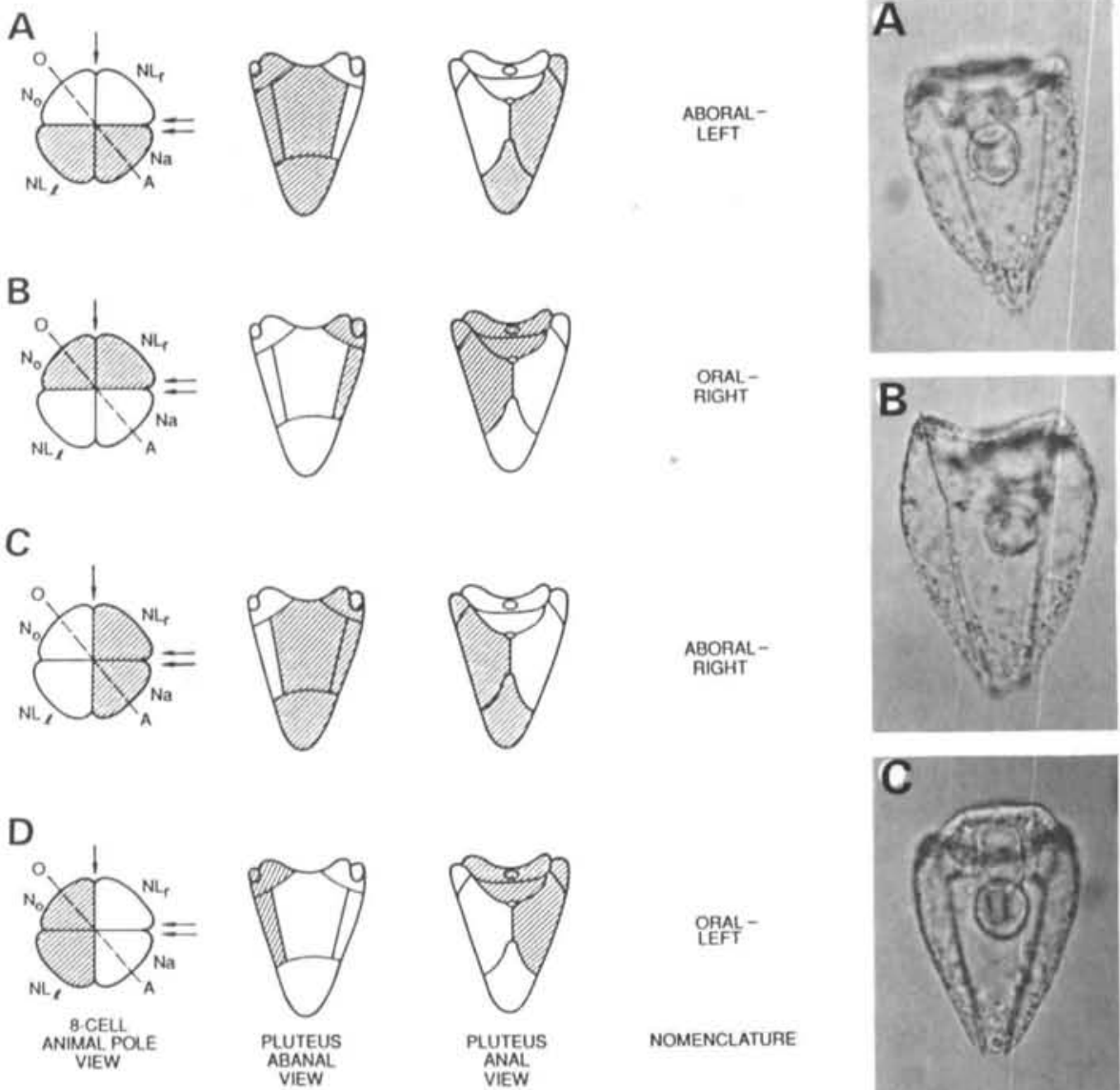

OPAL-

NOMENCLATURE
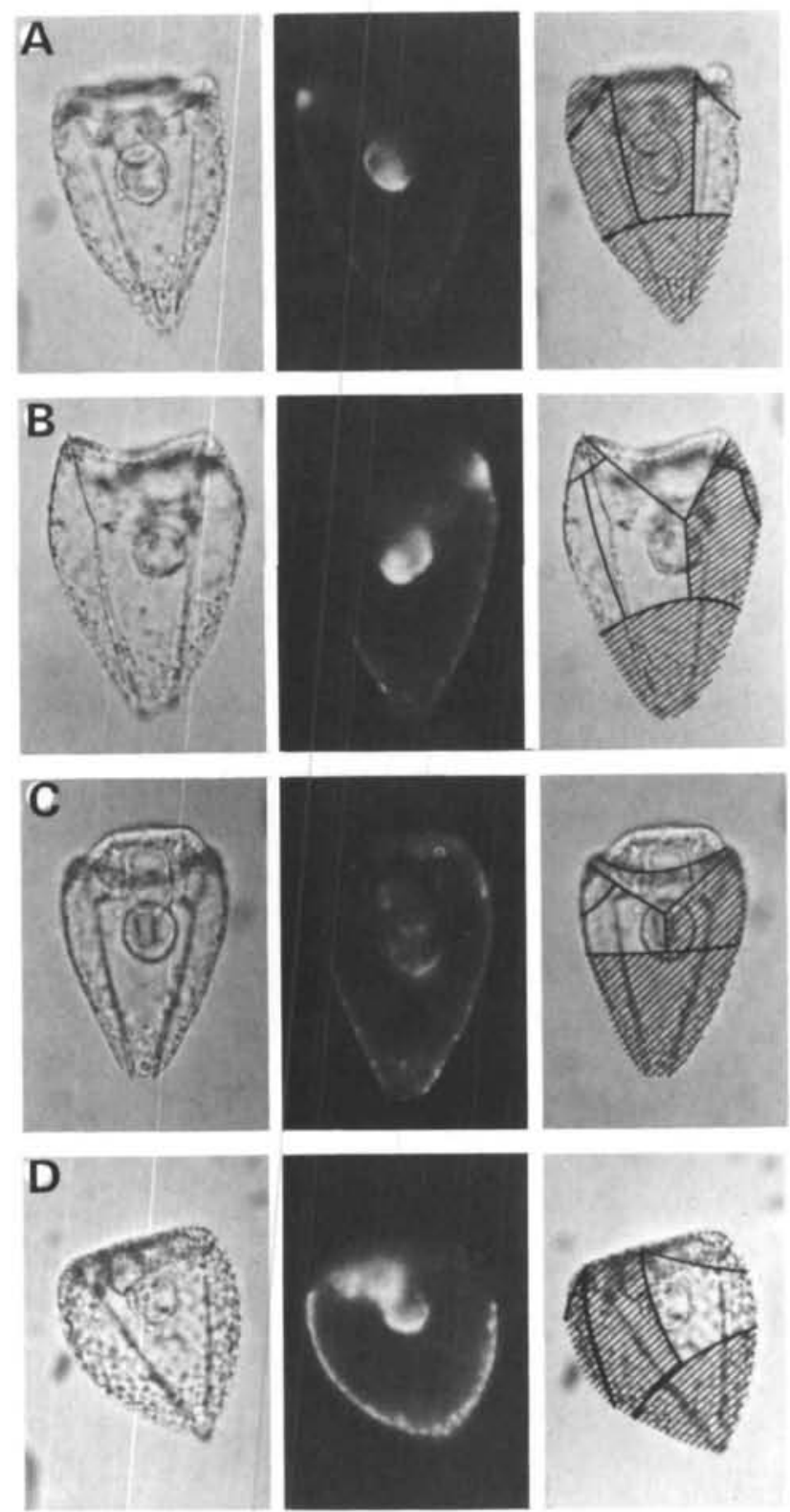

Fig. 2. Examples of four different plutei in which the 2-cell blastomere producing the $\mathbf{N a}, \mathbf{V A}, \mathbf{N L}_{\mathbf{L}}$ and $\mathbf{V L}_{\mathrm{L}}$ cells was injected. The left column depicts a bright-field view of the pluteus, the middle column is an epifluorescent image and the right column displays the tracts of cells labeled (hatched), as determined by observations of many different focal planes. The focus shown is approximately medial, in depth, in order to best illustrate the crucial labeled cells. (A) Abanal view. The plane of focus lies on the nether surface of the pluteus, away from the viewer. $(B, C)$ Anal view. (D) Abanal view.

that in a photograph adequately illustrates the pattern of labeling in the curved, single-cell-thick ectodermal surface. Thus in Fig. 2, for example, the focal plane shown illustrates the asymmetry of ectodermal labeling of a focal level midway through the embryo, and the remaining positions of these patterns, illustrated by the 
superimposed striping in the right column of this figure, were established by manually changing the plane of focus.

Fig. 2 illustrates the aboral-left pattern, i.e. that predicted in Fig. 1A. In embryos displaying this pattern, the apical, midregion, left lateral anterior and left anal plate tracts of the aboral ectoderm display fluorescent label. When viewed from the anal surface at a medial plane of focus as in Fig. 2B,C, a distinctive indicator of this pattern is fluorescent signal in all of the aboral ectoderm except for an anterior region on the right flank of the embryo (right and left are considered from the point of view of the embryo, not the observer). The facial ectoderm around the mouth and the ciliated band are conspicuously dark. When viewed from the abanal side as in Fig. 2A,D, the dark right flank of the embryo is on the viewer's right, and the remainder of the thin aboral ectoderm wall is labeled. Similarly, the facial ectoderm around the mouth and the ciliated band are unlabeled. This pattern results when the 8-cell blastomeres $\mathbf{N a}, \mathbf{V A}, \mathbf{N L}_{\mathbf{L}}$ and $\mathbf{V L}_{\mathbf{L}}$ all descend from the injected 2-cell blastomere (Fig. 1A).

The complementary pattern, in which the oral ectoderm, supra-anal ectoderm, right anal plate aboral ectoderm and right lateral aboral ectoderm contained fluorescent dye is illustrated in Fig. 3. A prominent feature of this labeling pattern is a high level of fluorescent signal in the facial ectoderm and ciliated band, which obscures the microscopic details of these tissues. This condition results from the structure of the ciliated band, which consists of closely stacked columnar cells that when labeled with the fluorescent dye produce high emissions in a small volume. The most direct diagnostic feature of this pattern is the presence or absence of dye in the lateral and anal plate ectoderm. When viewed from the abanal side at a surface plane of focus as in Fig. 3A, this tract of cells can be seen on the embryo's right (the viewer's right) just posterior to the highly fluorescent supra-anal ectoderm, ciliated band and gut. When viewed from the anal side (Fig. 3B,C), the right anal plate ectoderm appears on the viewer's left posterior to the supra-anal ectoderm. As shown in Fig. 1B, this pattern must result from labeling of the first cleavage blastomere that gives rise to the oral quadrant plus the right lateral cells, i.e. the 8-cell-stage blastomeres No, VO, $\mathbf{N L}_{\mathbf{R}}$, and $\mathbf{V L}_{\mathbf{R}}$.

The converse associations, viz. the progeny of the oral quadrant with those of the left lateral one (No, VO, $\mathbf{N L}_{\mathrm{L}}$ and $\mathbf{V L}_{\mathbf{L}}$ derivatives, Fig. 1D); or the progeny of the aboral quadrant with those of the right lateral one $\left(\mathbf{N a}, \mathbf{V A}, \mathbf{N L}_{\mathbf{R}}\right.$ and $\mathbf{V L}_{\mathbf{R}}$ derivatives, Fig. 1C), were also observed, though as discussed below, very infrequently. The three embryos found that displayed the aboralright pattern resemble those illustrated in Fig. 1A, except for their opposite labeling patterns. Though only two possible cases were found, the oral-left pattern (Fig. 1C) also occurs. However, these cases were difficult to analyze because of very low label intensity in the oral regions. These examples are less certain; nonetheless, because they were not noticeably abnormal in morphology they are included in the following analysis.
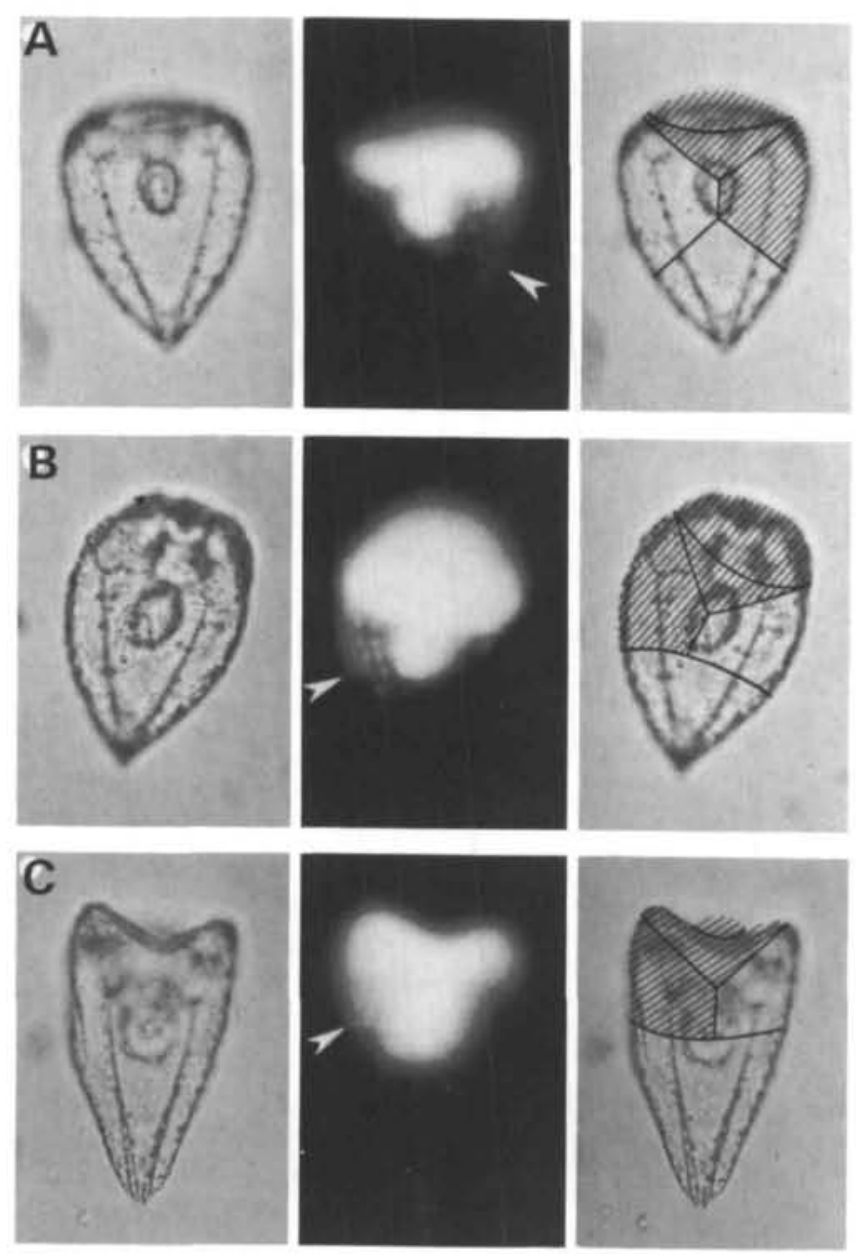

Fig. 3. Examples of three different plutei in which the 2-cell blastomere producing $\mathrm{No}, \mathrm{VO}, \mathbf{N L}_{\mathbf{R}}, \mathbf{V L}_{\mathbf{R}}$ cells was injected. The left column depicts a bright-field view of the pluteus, the middle column is an epifluorescent image and the right column displays the tracts of cells labeled, as in Fig. 2. The surface of the anal ectoderm is in the plane of focus to best illustrate the diagnostic labeled cells.

(A) Abanal view. (B,C). Anal view.

Table 1. Labeling patterns in pluteus-stage embryos injected in a single blastomere with lysyl rhodamine-dextran at the 2-cell stage

\begin{tabular}{lccccc}
\hline $\begin{array}{l}\text { Pattern } \\
\text { observed* }\end{array}$ & Exp. 1 & Exp. 2 & Exp. 3 & Exp. 4 & Total \\
\hline Aboral-left & 2 & 7 & 13 & 5 & 27 \\
Oral-right & 1 & 1 & 14 & 4 & 20 \\
Aboral-right & 0 & 0 & 2 & 1 & 3 \\
Oral-left & 0 & 0 & 1 & 1 & 2
\end{tabular}

* See Fig. 1 and text for identification of patterns.

\section{Distribution of the observed labeling patterns}

A total of 52 normally developing pluteus stage embryos deriving from four different batches of eggs labeled at the 2-cell stage were scored. As shown in Table 1, the aboral-left pattern and its complement, the 

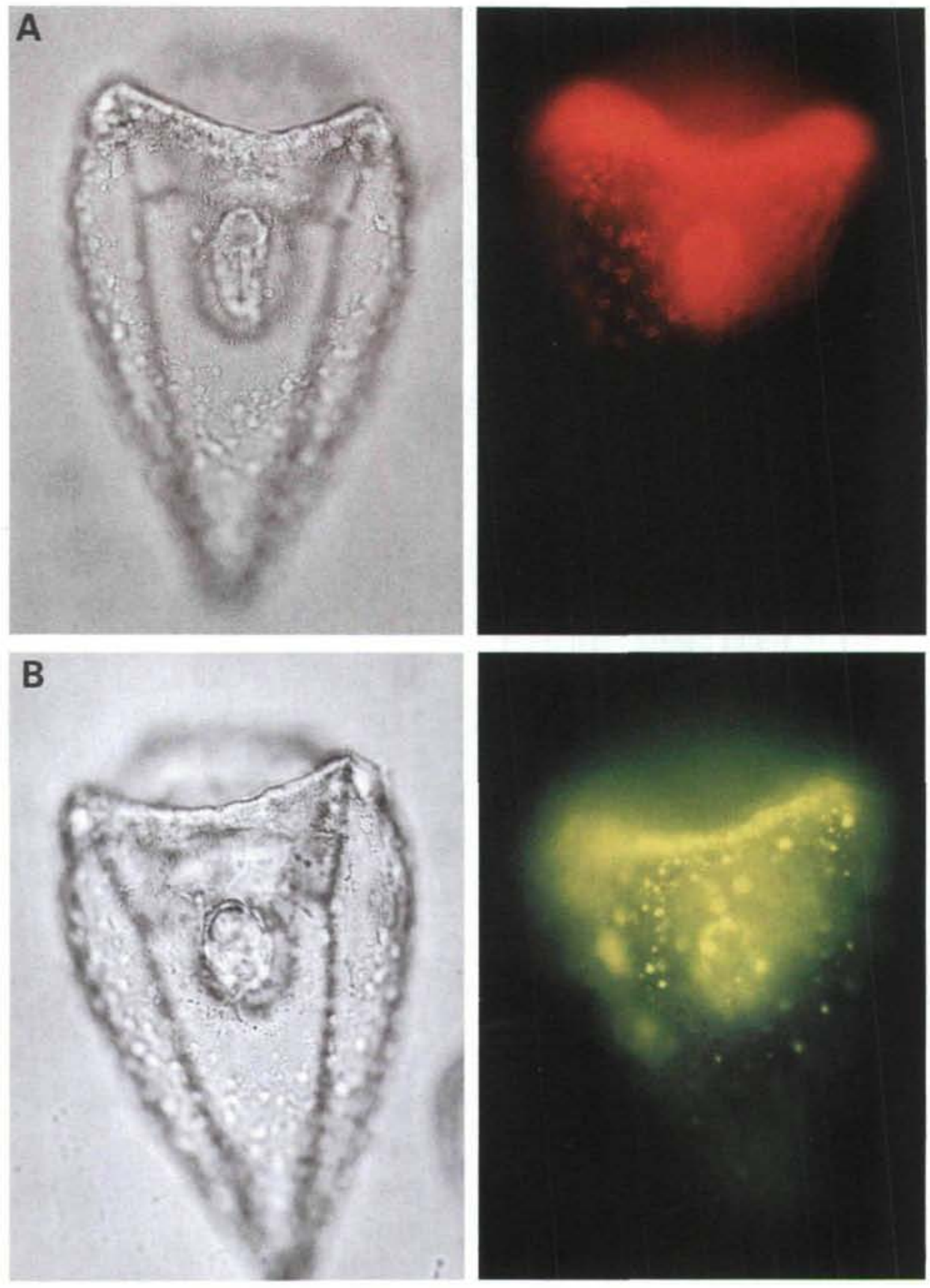

Fig. 4. Comparison of embryos injected with either Lucifer Yellow $\mathrm{CH}$ or lysyl rhodamine dextran. (A) A pluteus injected with rhodamine dextran in the oral/right half embryo (No, VO, NL $\mathbf{R}, \mathbf{V L}_{\mathbf{R}}$ ) and viewed from the anal side. Fluorescent signal is evident in the oral ectoderm, especially the ciliated band, the supra-anal ectoderm, and the right anal plate aboral ectoderm. (B) A pluteus injected with Lucifer Yellow $\mathrm{CH}$ for which no unambiguous assignment of pattern can be made. The fluorescence is punctate and apparently distributed in both left and right anal plate aboral ectoderms, and in the oral ectoderm, but not the apical aboral ectoderm. This was a typical example, and in our hands pluteus-stage embryos with this tracer were always difficult to interpret. 
oral-right pattern, occurred in 47 out of 52 cases and, as would be expected, similar numbers of each of these patterns were observed. As noted above, there were three examples of the aboral-right and two possible examples of the oral-left patterns. Thus the association of the descendants of the initial aboral quadrant $(\mathrm{Na}+\mathrm{VA})$ with those of the left lateral quadrant $\left(\mathbf{N L}_{\mathbf{L}}+\mathbf{V L}_{\mathbf{L}}\right)$, and of the initial oral quadrant $\left(\mathbf{N o}+\mathbf{V L}_{\mathbf{R}}\right)$ with the right lateral quadrant $\left(\mathbf{N L}_{\mathbf{R}}+\mathbf{V L}_{\mathbf{R}}\right)$ is at least nine times more prevalent than the opposite association (assuming that the rare patterns observed are in fact a normal, genuine occurrence, albeit of low frequency). The distribution shown in Table 1 is significantly different from that expected if $\mathrm{O}-\mathrm{Ab}$ axis specification were random with respect to quadrant $(P<0.001$ by Chisquare analysis; Snedecor \& Cochran, 1979).

\section{Lucifer Yellow injection results}

Recently the fluorescent dye Lucifer Yellow $\mathrm{CH}$ was used to trace cell lineage in a study of $\mathrm{O}-\mathrm{Ab}$ axis specification in embryos of Hemicentrotus pulcherrimus (Kominami, 1988). We also used Lucifer Yellow $\mathrm{CH}$ injections at the 2-cell-stage in $S$. purpuratus embryos, so as to be able to compare the results with those obtained using the rhodamine dextran derivative. As expected, when Lucifer Yellow $\mathrm{CH}$ is injected into Strongylocentrotus purpuratus embryos at the 2-cell stage, it homogeneously diffuses throughout the cell and remains evenly distributed at least until morula stage $(12 \mathrm{~h})$, clearly labeling half of the embryo cells. However, at later stages a different kind of pattern is observed. By $72 \mathrm{~h}$ the plutei exhibit a punctate rather than even distribution of Lucifer Yellow fluorescence that appears to result from sequestration of the tracer into some intracellular compartment, as illustrated in Fig. 4B. This makes it very difficult to determine whether a given group of cells is labeled or not, since a given cell may contain only one or two bright granules and its neighbor none. The example shown in Fig. 4B appears to be labeled in the oral ectoderm, but a few spots of dye can also be observed on both sides of the anus, i.e. in the right as well as the left lateral anal plate ectoderm. Thus, it is impossible to assign this example to either oral-right or oral-left patterns. In contrast, the matched dextran labeled example shown in Fig. 4A, which is heavily labeled in the oral ectoderm, clearly exhibits the dextran lineage tracer in the right lateral anal plate ectoderm, and not at all in the left lateral anal plate ectoderm.

\section{Discussion}

The oral-aboral axis is specified by the time of first cleavage

These results demonstrate unambiguously a high correlation between the position of the plane of first cleavage and the position of the $\mathrm{O}-\mathrm{Ab}$ axis. In at least $90 \%$ of cases, the axis forms about $45^{\circ}$ clockwise from the plane of first cleavage, as viewed from the animal pole, and is oriented so that by the 8-cell stage the first cleavage furrow separates the animal oral ectoderm progenitor cell No from the left lateral animal blastomere $\mathbf{N L}_{\mathbf{L}}$. The direction of causality in this process cannot be determined from these results; i.e. we cannot distinguish whether an $\mathrm{O}-\mathrm{Ab}$ polarization along the future axis follows from the establishment of the initial cleavage plane; or vice versa; or whether, as we think most probable (see below), both result from a prior orienting event. In any case, however, it would appear unavoidable that the process by which the orientation and location of the $\mathrm{O}-\mathrm{Ab}$ axis are specified has (in undisturbed embryos) taken place by the time first cleavage is complete. This specification remains labile, and the axis can be reorganized if the embryos are subdivided or recombined (see Introduction). Conceivably, only the first cleavage plane is determined at this early stage, and this plane is somehow marked. The actual physical processes leading to $\mathrm{O}-\mathrm{Ab}$ axial polarization could then occur slightly later with reference to the marked plane, though still prior to third cleavage. Our results show in any case that by first cleavage something has happened to the spatial organization of the egg cytoplasm in the plane orthogonal to the $\mathrm{A}-\mathrm{V}$ axis, which, in at least $90 \%$ of cases, directly forecasts the $\mathrm{O}-\mathrm{Ab}$ axis and the consequent larval plane of bilateral symmetry. Nor in the other $10 \%$ (or less) is the axis random with respect to the plane of first cleavage. In this minor class of embryos, the orientation is a mirror image of the geometry of the dominant class, so that the axis appears $45^{\circ}$ counterclockwise from the cleavage furrow. This could be due to an innate (i.e. genetically controlled) reversal of chirality in some cytostructural component or, for example, could reflect a $10 \%$ ratio of probabilities of two competing forms of cytostructural assemblage. Thus, in both clockwise and counterclockwise forms, there is an unmistakeable spatial relation between first cleavage and $\mathrm{O}-\mathrm{Ab}$ axis specification. These results are consistent with those of our earlier study (Cameron et al. 1987), as well as with the observation of Czihak (1963), since both of these indicate that $\mathrm{O}-\mathrm{Ab}$ axial specification has taken place prior to third cleavage. Classical workers, who carried out various vital staining experiments designed to approach this issue, saw that the plane of first cleavage itself does not correspond with the plane of bilateral symmetry (reviewed by Hörstadius, 1973, p. 93), but they missed the relation we have shown here. This they could not easily have perceived, however, for until the recent studies of Cameron et al. (1987) the lineage of the embryo ectoderm was incorrectly assumed and, as we illustrate in the present work, this knowledge is required for an adequate analysis.

The conclusion drawn here is in conflict with results reported by Kominami (1988) in a recent study carried out on Hemicentrotus pulcherrimus. This author deduced that $\mathrm{O}-\mathrm{Ab}$ specification occurs only after 5-6th cleavage, on the basis that eight different patterns of labeling are observed with respect to this axis when Lucifer Yellow $\mathrm{CH}$ is injected as a lineage tracer into first cleavage embryos; meridional boundaries are first aligned into eight separate rows at the 5-6th cleavage. 
While it is certainly possible that in $H$. pulcherrimus O-Ab specification takes place differently than in $S$. purpuratus, there is in our opinion another possible explanation for the discrepancy. Our own direct comparison of Lucifer Yellow CH with the higher molecular weight fluorescent dextrans used here and in the previous study (Cameron et al. 1987) lead us to the conclusion that Lucifer Yellow is an unsatisfactory lineage tracer. Fig. 4 of this paper demonstrates the ambiguity that results from the punctate distribution obtained with the Lucifer Yellow $\mathrm{CH}$ tracer. One possible feature that could contribute ambiguity in the pattern of Lucifer Yellow labelling is that this dye can pass through gap junctions (Bennett \& Goodenough, 1978).

\section{Possible mechanism of $O-A b$ axis specification}

We assume in the following that the $\mathrm{O}-\mathrm{Ab}$ axis comes into being after fertilization, and that the unfertilized egg is radially symmetric, as are other deuterostome eggs. There is no significant evidence contrary to this assumption. Thus the $\mathrm{O}-\mathrm{Ab}$ axis is established with reference to the first cleavage plane in the course of the extensive cytoskeletal reorganization that follows fertilization. The cleavage furrow forms in the plane that bisects the mitotic apparatus and this, in turn, is established by the position of the centrosomes (reviewed by Rappaport, 1971). In sea urchins, both centrosomes are brought into the zygote by the fertilizing sperm (Paweletz et al. 1987a,b). One centrosome remains firmly attached to the sperm pronuclear membrane, while the other migrates around the pronuclei with the sperm mitochondrion until it reaches a position opposite the attached one. The attached centrosome, called the 'stationary' one (Paweletz et al. 1987a,b), stays in place throughout the period of pronuclear fusion and is the earliest known feature imparting a horizontal asymmetry to the zygote. This unique 'stationary' centrosome could serve to nucleate the polarized cytoskeletal organization that initially specifies the $\mathrm{O}-\mathrm{Ab}$ axis, and could identify the quadrant of the embryo that ultimately forms one pole of this axis, i.e. either oral or aboral. One consequence of this cytoplasmic polarization would be the concentration of redox (mitochondrial?) enzymes at the oral pole noticed by Czihak (1963). Both the specification of the $\mathrm{O}-\mathrm{Ab}$ axis and the location of the plane of first cleavage would result in turn from the rotational positions occupied by the sperm centrosomes. Since the sperm pronuclei describe various paths into the egg, rotating as they traverse the cytoplasm (Schatten, 1982), a simple relation between the point of sperm entry per se and the location of either the cleavage plane or the $\mathrm{O}-\mathrm{Ab}$ axis would not be expected, and such a relation is not observed.

Parthenogenic sea urchin eggs develop with fairly good efficiency (Ishikawa, 1975; Loeb, 1914). Thus even subtle asymmetries might provide default orienting cues for the cytoskeletal rearrangements involved in the initiation and positioning of the cleavage planes and in $\mathrm{O}-\mathrm{Ab}$ axial polarization. This should not seem surprising in view of the clear demonstration that in amphibian eggs gravity-driven rotation, for example, can provide an alternate spatial cue overriding the rotation normally promoted by sperm entry (Gerhart et al. 1981). Furthermore, in regulative reestablishment of the $\mathrm{O}-\mathrm{Ab}$ axis in egg fragments and egg and embryo fusions alternative spatial cues are also likely to be utilized. Thus, the picture that emerges is of a balanced but very labile, radially symmetric, cytoplasmic system within which, on presentation of some internal asymmetry that for want of a better term we think of as a nucleation center, there will coalesce two structures, by a related process. These are the initial mitotic apparatus, and to the counterclockwise side of this, the $\mathrm{O}-\mathrm{Ab}$ polarization. From such delicately poised beginnings there subsequently derive the organized arrays of differentially functioning cells which by late cleavage already distinguish the oral and aboral ectoderm precursors.

Research was supported by NIH Grant HD-05753 (to E.H.D.) and by NSF Grant BNS8608356 and a McKnight Scholar Award (to S.E.F.). The manuscript improved through the helpful criticism of our colleagues, Drs B. HoughEvans, E. Rothenberg and R. Whittaker.

\section{References}

Bennett, M. V. L. \& Goodenough, D. A. (1978). Nonelectrical functions of gap junctions. Neurosci. Res. Program Bull. 16, 4434-4449.

Boveri, T. (1901). Die Polaritat von Oocyte, Ei und Larve des Strongylocentrotus lividus. Zool Jahrb., Abt. Anat. Ontog. Tiere. 14, 630-653.

Cameron, R. A., Fraser, S. E., Britten, R. J. \& Davidson, E $H$. (1989). The progeny of lateral quadrants of the sea urchin egg segregate exclusively to aboral ectoderm at the 5 th cleavage. Devl Biol., submitted for publication.

Cameron, R. A., Hough-Evans, B. R., Britten, R. J. \& Davidson, E. H. (1987). Lineage and fate of each blastomere of the eight-cell sea urchin embryo. Genes \& Dev. 1, 75-84.

CzlHAK, G. (1963). Entwicklungsphysiologische Untersuchungen an Echiniden (Verteilung und Bedeutung der Cytochromoxydase). Wilhelm Roux's Archiv. EntwickMech. Org. 154, 272-292.

Gerhart, J., Ubbels, G., Black, S., Hara, K. \& Kirschner, M. (1981). A reinvestigation of the role of the grey crescent in axis formation in Xenopus laevis. Nature, Land. 292, 511-516.

Horstadius, S. (1973). Experimental Embryology of Echinoderms. Clarendon Press, Oxford.

Ishikawa, M. (1975). Parthenogenetic activation and development. In The Sea Urchin Embryo (ed. G. Czihak), Berlin: SpringerVerlag, pp. 148-169.

Kominami, T. (1988). Determination of dorso-ventral axis in early embryos of the sea urchin, Hemicentrotus pulcherrimus. Devl Biol. 127, 187-196.

LOEB, J. (1914). On the nature of the process of fertilization and the artificial production of normal larvae (plutei) from the unfertilized eggs of the sea urchin. Am. J. Physiol. 3, 135-138.

McMahon, A. P., Flytzanis, C. N., Hough-Evans, B. R.

Katula, K. S., Britten, R. J. \& Davidson, E. H. (1985) Introduction of cloned DNA into sea urchin egg cytoplasm: Replication and persistence during embryogenesis. Devl Biol. 108, 420-430.

PaWeletz, N., Mazia, D. \& Finze, E.-M. (1987a). Fine structural studies of the polarization of the mitotic apparatus in the fertilized sea urchin egg. I. The structure and behavior of centrosomes before fusion of the pronuclei. Eur. J. Cell Bıol. 44 , $195-204$.

Paweletz, N., Mazia, D. \& Finze, E.-M. (1987b). Fine structural 
studies of the bipolarization of the mitotic apparatus in the fertilized sea urchin egg. II. Bipolarization before the first mitosis. Eur. J. Cell Biol. 44, 205-213.

Rappaport, R. (1971). Cytokinesis in animal cells. Intl. Rev. Cytol. 31, 169-213.

SARdet, C. \& Chang, P. (1985). A marker of animal-vegetal polarity in the egg of the sea urchin Paracentrotus lividus. The pigment band. Expl Cell Res. 160, 73-82.

Schatten, G. (1982). Motility during fertilization. Intl. Rev. Cytol. 79, 59-163.
SCHROEder, T. (1980a). Expressions of the prefertilization polar axis in sea urchin eggs. Devl Biol. 79, 428-443.

SCHroeder, T. (1980b). The jelly canal: marker of polarity for sea urchin oocytes, eggs, and embryos. Expl Cell Res. 128, 490-494.

Snedecor, G. W. \& Cochran, W. G. (1979). Statistical Methods, Iowa State University Press, Ames, Iowa. 